\title{
RELAÇÃO ENTRE ESTRESSE E HABILIDADES SOCIAIS EM DOCENTES DO ENSINO SUPERIOR DO ESTADO DA BAHIA
}

RELATIONSHIP BETWEEN STRESS AND SOCIAL SKILLS IN PROFESSORS IN THE STATE OF BAHIA

\section{RELACIÓN ENTRE ESTRÉS Y HABILIDADES SOCIALES EN LOS DOCENTES UNIVERSITARIOS DEL ESTADO DE BAHÍA}

\author{
Adriana Cristina Boulhoça Suehiro* \\ Larissa Muniz R. A. dos Santos**
}

\begin{abstract}
RESUMO
Este estudo pretendeu caracterizar o estresse e as habilidades sociais de docentes do ensino superior do Estado da Bahia. Ao lado disso, buscou verificar a relação existente entre esses construtos. Para tanto, aplicou o Inventário de Sintomas de Stress para Adultos de Lipp e o Inventário de Habilidades Sociais (IHS) em 152 docentes do ensino superior de instituições públicas e privadas da Bahia. Os participantes se dedicavam a diferentes áreas e, em sua maioria, pertenciam ao gênero masculino. Trinta e cinco docentes apresentaram quadros característicos das fases de resistência e quase-exaustão. Ao lado disso, evidenciou-se que os sintomas físicos foram os mais presentes, assim como um baixo repertório de habilidades sociais em "autocontrole da agressividade" e "enfrentamento e autoafirmação com risco". Não houve diferenças significativas em habilidades sociais entre os docentes com sintomatologia de estresse e os sem sintomatologia. Dada a relevância da temática e suas implicações, ressalta-se a necessidade de novas investigações.
\end{abstract}

Palavras-chave: Sintomas de estresse. Competência social. Habilidades sociais. Ensino superior.

\section{ABSTRACT}

This study sought to characterize the stress and social skills of professors in higher education in the state of Bahia. We attempted to verify the relationship between these constructs. To do so, it has been applied the Symptoms of Stress Inventory by Lipp and Social Skills Inventory (HSI) in

\footnotetext{
Texto recebido em 30 de abril de 2014 e aprovado para publicação em 25 de fevereiro de 2016.

Pós-Doutora em Educação pela Universidade Estadual de Campinas (apoio CNPq), doutora em Psicologia pela Universidade São Francisco, docente da Universidade Federal do Recôncavo da Bahia (UFRB)/Santo Antônio de Jesus, líder do Laboratório de Instrumentação e Avaliação Psicológica da UFRB (Labiap), psicóloga. E-mail: dricbs@yahoo.com.br.

* Mestra em Gestão de Políticas Públicas pela UFRB, coordenadora do Serviço de Psicologia da UFRB, membro do grupo de pesquisa do Laboratório de Instrumentação e Avaliação Psicológica da UFRB (Labiap), psicóloga. E-mail: lara.muniz@hotmail. com.
} 
152 professors in higher education public and private institutions of Bahia. Participants were engaged in different areas and, in most cases, were male. Thirty-five professors showed characteristic frames phases of resistance and near-exhaustion. In addition, it became clear that the physical symptoms were more present, as well as low social skills in "Self-Control Aggression" and "Coping with risk and self-assertion". There were no significant differences in social skills among Professors with symptoms of stress and Professors without symptoms. Given the relevance of this topic and its implications, it is worth highlighting the need for further investigations.

Keywords: Symptoms of stress. Social competence. Social skills. Higher education.

\section{RESUMEN}

Este estudio trata de caracterizar el estrés y las habilidades sociales de los docentes de la educación superior en el estado de Bahía. Junto a esto, se intentó verificar la relación entre estos constructos. Para ello, se aplicó el Inventario de Síntomas de Estrés para Adultos de Lipp y el Inventario de Habilidades Sociales (IHS) en 152 docentes de las instituciones públicas y privadas de educación superior de Bahía. Los participantes se dedicaban a diferentes áreas y, en la mayoría de los casos, pertenecían al género masculino. Treinta y cinco docentes mostraron marcos característicos de las fases de resistencia y agotamiento casi total. Junto a esto, se hizo evidente que los síntomas físicos fueron los más presentes, así como un bajo repertorio de habilidades sociales en "autocontrol de la agresividad" y "enfrentamiento y autoafirmación con riesgo". No hubo diferencias significativas en habilidades sociales entre los docentes con síntomas de estrés y los que no tenían esos síntomas. Dada la relevancia de este tema y sus implicaciones, se destaca la necesidad de nuevas investigaciones.

Palabras clave: Síntomas de estrés. Competencia social. Habilidades sociales. Educación superior.

\section{INTRODUÇÃO}

0 estresse no trabalho tem sido considerado como um objeto de preocupação e reflexão por diversos estudiosos, sobretudo devido às graves consequências à saúde e ao desempenho dos trabalhadores (Costa \& Ferreira, 2014; Minari \& Souza, 2011; Sadir \& Lipp, 2013; Tamayo, 2001). São concebidas como situações de estresse laboral aquelas em que o indivíduo desenvolve uma percepção do seu ambiente de trabalho como ameaçador, decorrente de demandas ambientais excessivas para as quais ele não tem recursos adequados de 
enfrentamento. Para Segantin e Maia (2007), o tipo de desgaste a que as pessoas são expostas nos ambientes e as relações com o trabalho são fatores determinantes de doenças e transtornos relacionados ao estresse, o que pode gerar impactos sobre sua saúde física e mental. Em termos de implicações físicas, os sinais e sintomas que ocorrem com maior frequência são o aumento da sudorese, tensão muscular, taquicardia, hipertensão, aperto da mandíbula, ranger de dentes, hiperatividade, náuseas, mãos e pés frios (Lipp, 2000; Sadir \& Lipp, 2013). Já em relação à saúde mental, estudos demonstram que a ocorrência de estresse em níveis elevados, pode resultar em prejuízos ao funcionamento adequado do aparelho psíquico, contribuindo para vários desfechos, como burnout, transtornos mentais comuns e morbidades mentais mais graves (Borine, Assis, Lopes, \& Santini, 2012; Fernandes, 1999; Tironi et al., 2009).

Segundo Sadir, Bignotto, e Lipp (2010), o estresse tem diferentes etiologias e pode se desenvolver tanto devido à necessidade de lidar com fatores externos ao organismo e capazes de gerar tensões quanto diante de condições internas capazes de atuar como geradoras de estados tensionais significativos. Nesse sentido, Takahashi (2009) aponta que os eventos estressores são aqueles que geram respostas de estresse e demandam uma adaptação do indivíduo a eles exposto. Tais eventos podem ser considerados externos, quando são provenientes de fontes que, na maioria das vezes, estão fora do controle do indivíduo, como mortes, violência urbana, acontecimentos de vida e demissão; e internos, quando englobam agentes que vêm do interior, como o padrão de comportamento, pensamentos, crenças e valores pessoais (Christophoro \& Waidman, 2002). Assim, diversos estudos (Bachion, Abreu, Godoy, \& Costa, 2005; Christophoro \&Waidman, 2002; Esteves, 2004; Lipp, 2008; Malagris \& Fiorito, 2007; Oliveira \& Cardoso, 2011; Pereira, Amaral, \& Scorsolini-Comin, 2011; Reinhold, 1996; Reis, Carvalho, Araújo, Porto, \& Silvany Neto, 2005; Servilha, 2005; Takahashi, 2009) têm se proposto a investigar as reações de estresse e os eventos estressores em diferentes categorias profissionais, inclusive entre professores universitários. De maneira geral, essas pesquisas têm apontado que o excesso de trabalhos burocráticos e salário baixo estão dentre os principais eventos estressores apontados pelos professores avaliados, assim como a superlotação de alunos nas salas de aula, o trabalho com estudantes desinteressados, indisciplinados e dispersivos, o pequeno número de faltas abonadas, as condições de trabalho, a dupla ou tripla jornada, as questôes financeiras e as pressões do trabalho, como pós-graduação, competitividade e relacionamento interpessoal conflitante.

Buscando confirmar o fato de que a docência se constitui como uma profissão exposta a condiçôes estressoras, ao focalizar a relação estresse e trabalho docente, Servilha (2005), por exemplo, investigou a presença de estresse em 23 professoras 
universitárias, da área de Fonoaudiologia, de uma universidade particular. Verificou que os sintomas de estresse, tal como mensurados pelo Inventário de Sintomas de Stress para Adultos de Lipp (ISSL) (Lipp, 2000), foram assinalados por $47,82 \%$ das docentes, casadas e na faixa etária de 40 a 49 anos. A fase predominante foi a de resistência, e os sintomas mais encontrados foram os físicos, evidenciando que é muito mais o corpo do professor que expressa as suas tensões emocionais do que a sua cognição.

Mais recentemente, Pereira, Amaral, e Scorsolini-Comin (2011) avaliou a presença de sintomas de estresse em uma amostra de professores de uma universidade privada do interior do Estado de Minas Gerais. Participaram do estudo 147 professores de diferentes cursos das áreas de Humanas, Saúde, Educação, Tecnológica e Veterinária. Foi usado o ISSL. Os resultados encontrados indicaram que $55,1 \%$ dos professores não mostraram sintomas de estresse. Para os demais (44,9\%), o estresse se apresentou em grau moderado, estando a maioria na fase de resistência. Foi possível verificar, ainda, que os sintomas psicológicos apareceram em porcentagem maior que os físicos e, em alguns casos, associados a estes.

Oliveira e Cardoso (2011) também realizaram um estudo com o objetivo de investigar as manifestaçôes clínicas de estresse e os fatores estressantes no trabalho de docentes da área de saúde de uma universidade pública do Norte do Brasil. As autoras utilizaram como instrumentos da referida pesquisa o ISSL e um questionário (Santos \& Cardoso, 2010) que contém itens sobre o perfil sociodemográfico e sobre a percepção do próprio estresse. A amostra do estudo foi composta por 91 docentes e, como resultados, Oliveira e Cardoso (2011) apontaram que foi possível verificar que 75,8\% dos participantes não apresentaram manifestações clínicas de estresse, e dos 24,2\% que apresentaram tais manifestações, 95,4\% encontravam-se na fase de resistência. Em relação à sintomatologia, verificou-se que 63,6\% apresentavam sintomas físicos, e 36,4\%, sintomas psicológicos. Quanto à percepção do próprio estresse, do estresse no trabalho e das condiçôes de trabalho, os docentes avaliaram-se como muito estressados. Os resultados referentes às manifestaçôes clínicas de estresse estiveram significativamente associados aos docentes com filhos e que se percebem como muito estressados.

Estudos como esses levantam a necessidade de se realizarem novas pesquisas acerca de uma importante questão de saúde pública que é o estresse no trabalho de diferentes categorias profissionais, bem como investigar a relação entre esse construto e outros aspectos, como o repertório de habilidades sociais do trabalhador. As habilidades sociais podem ser entendidas como comportamentos que capacitam a pessoa a interagir com o outro de modo que as suas necessidades 
sejam satisfeitas e satisfaçam às expectativas dos demais. Esses comportamentos incluem não somente a verbalização como também a expressão facial, a postura, o contato visual, gestos, aparência física, entre outros. Nessa perspectiva, o indivíduo socialmente competente é visto como autorreforçador e, portanto, com uma boa autoestima e com um bom senso de autoeficácia, o que facilita seu convívio com as dificuldades e o seu enfrentamento diante de eventos estressantes (Lucca, 2004; Maia \& Bertolini, 2012; Maia \& Lobo, 2013; Pureza, Rursch, Wagner, \& Oliveira, 2012). Assim, as habilidades sociais podem ser consideradas como imprescindíveis para o desenvolvimento socioemocional e o ajustamento do indivíduo.

Entre os estudos realizados com professores estão os de Soares e Melo (2010) e Soares, Gomes, e Prata (2011). As primeiras autoras buscaram comparar as habilidades sociais de docentes de diferentes segmentos do ensino com as de outros profissionais. Para tanto, avaliaram 527 participantes, sendo 264 professores do nível infantil ao nível superior de ensino e 263 não professores. Os resultados encontrados por Soares e Melo (2010) sugerem que, em geral, os docentes apresentam repertórios mais elaborados de habilidades sociais, quando comparados aos profissionais não professores. Ao lado disso, indicaram diferença significativa entre os grupos, sendo que os docentes obtiveram médias superiores aos demais em autoafirmação na expressão de afeto positivo, autoexposição a desconhecidos ou a situações novas e na pontuação total no IHS (Del Prette \& Del Prette, 2001).

Soares, Gomes, e Prata (2011) também compararam os repertórios de habilidades sociais dos profissionais professores e não professores, no entanto, dessa vez, considerando suas áreas de atuação. Participaram do estudo 527 profissionais de ambos os gêneros. Entre os docentes, $42,42 \%$ eram da área de Ciências Humanas; 15,53\% de Ciências Naturais; 13,64\% de Ciências Exatas e 13,26\% de Letras e Artes. Já entre os profissionais não professores, 15,97\% pertenciam à área de Ciências Humanas; 15,97\%, às Ciências Exatas; 16,35\%, às Letras e Artes; 18,25\%, às Ciências Biológicas; 14,45\%, às Ciências Agrárias; e 19,01\%, às Ciências Sociais Aplicadas.

Os resultados evidenciaram que não houve diferenças de habilidades sociais entre docentes de diferentes áreas de atuação. Entre os profissionais não professores, os mais habilidosos foram os das Ciências Agrárias para enfrentamento com risco, conversação e desenvoltura social e, os de Ciências Humanas, em autoexposição a desconhecidos ou a situações novas. A comparação entre professores e não professores indicou que os profissionais das Ciências Agrárias são mais habilidosos que os demais em enfrentamento com risco. Em habilidade de autoafirmação na expressão do afeto positivo, conversação e desenvoltura social, autoexposição 
a desconhecidos ou a situações novas e na pontuação total, os professores de Ciências Humanas obtiveram melhor repertório em habilidades sociais.

A aprendizagem das habilidades sociais se constitui como um processo que ocorre "naturalmente" por meio das interações sociais que o indivíduo estabelece ao longo da vida (Del Prette \& Del Prette, 2010/2012; Del Prette, Del Prette, $\&$ Barreto, 2006). No entanto, quando essas interações são desfavoráveis, alguns déficits e problemas de competência social podem surgir e gerar consequências negativas no repertório de interações interpessoais e à qualidade de vida das pessoas. Ao lado disso, uma gama de estudos vem demonstrando que indivíduos com bons relacionamentos interpessoais tendem a serem mais saudáveis, menos propensos a doenças e também mais produtivos no trabalho (Bandeira, Rocha, Pires, Del Prette, \& Del Prette, 2006; Bighetti, 2010; Carneiro \& Falcone, 2013; Gomes et al., 2013; Gomide, Salvo, Pinheiro, \& Sabbag, 2005; Rockhill, Stoep, Mccauley, \& Katon, 2009). A despeito disso, poucas investigaçôes se detiveram na análise conjunta entre o estresse e o repertório de habilidades sociais (Grün, 2008; Pellegrini, Calais, \& Salgado, 2012) e somente um estudo abordou essa relação na população aqui focalizada, ou seja, docentes (Gomes et al., 2013).

Embora não tenham abordado professores, os estudos de Grün (2008) com 21 pacientes com câncer de laringe de um hospital de câncer de Curitiba, e Pellegrini, Calais, e Salgado (2012), com 83 universitários primeiranistas, mostraram que, de maneira geral, a maioria dos participantes ficou abaixo da média para habilidades sociais. Ao lado disso, Grün (2008) não constatou associações estatisticamente significativas entre estresse e habilidades sociais. Semelhantemente, os resultados de Pellegrini, Calais, e Salgado (2012) não apontaram relação estatística entre estresse e déficit em habilidades sociais, embora tenha havido correlação entre má administração de tempo e a presença de estresse.

Os resultados com professores não foram diferentes. O nível de significância obtido entre o escore total em habilidades sociais e o escore total em estresse foi 0,969. Gomes et al. (2013) investigaram as habilidades sociais e o nível de estresse de 65 professores do ensino fundamental atuantes em escolas públicas do Rio de Janeiro. Com relação ao nível de estresse, o ISSL mostrou que 23 $(35,3 \%)$ dos professores apresentaram estresse. Entre estes, a maioria $(n=1)$; $60,7 \%)$ encontra-se na fase de resistência, enquanto quatro $(0,6 \%)$ foram classificados na fase de exaustão. As análises estatísticas, quanto às habilidades sociais, mostraram que, por um lado, dos 23 professores que apresentam estresse: 8 foram identificados com repertório abaixo da média em habilidades sociais, sendo que 3 apresentaram indicação para treinamento. Por outro, 42 docentes não apresentaram estresse. Dentre esses 42, 11 foram identificados com repertório abaixo da média em habilidades sociais, dos quais 8 tinham indicação para treinamento nessas habilidades. 
Diantedoexposto, do número restrito de pesquisas que abordem conjuntamente estresse e habilidades sociais em docentes, da escassez de investigaçôes dessa natureza com professores universitários, assim como da relevância desses construtos para a qualidade de vida e produtividade dos envolvidos, este estudo pretendeu caracterizar o estresse e as habilidades sociais de docentes do ensino superior do Estado da Bahia. Ao lado disso, buscou verificar a relação existente entre esses construtos.

\section{MÉTODO}

\subsection{Participantes}

Participaram deste estudo 152 docentes do ensino superior, de ambos os gêneros, entre 24 e 65 anos $(M=40,36 ; \mathrm{DP}=8,34)$. A maioria dos participantes era do gênero masculino $(\mathrm{n}=82 ; 53,9 \%)$, sendo que 111 (73\%) eram de instituições públicas e 41 (27\%) de instituições particulares do Estado da Bahia. Entre os docentes que informaram a área de conhecimento à qual se dedicam, 61 (47,29\%) pertenciam às Ciências Humanas, 35 (27,13\%) às Ciências da Saúde e 33 (25,28\%) à área de Exatas.

\subsection{Instrumentos}

Inventário de Sintomas de Stress para Adultos de Lipp (ISSL) - É um instrumento que tem como finalidade identificar a sintomatologia apresentada pelo sujeito, avaliando a presença de sintomas de estresse, o tipo de sintoma (físico ou psicológico) apresentado e a fase em que o sujeito se encontra (Lipp, 2000). O inventário foi validado por meio de análise de consistência interna e análise fatorial, obtendo-se um alfa de Cronbach de 0,91 para a escala geral e dois eixos ou fatores, sendo que o primeiro incluía itens de natureza psicológica e o segundo, de natureza física. Assim, o ISSL ficou composto por 37 itens de natureza somática (física) e 19 psicológicos.

O manual do ISSL (Lipp, 2000) fornece o procedimento para se calcularem as porcentagens de sintomas de cada um dos três quadros que compõem sua avaliação e que representam quatro fases de estresse, e também de sintomas físicos e psicológicos. Nesta pesquisa, contudo, optou-se por não utilizar esse método de correção e sim fazer uso dos resultados brutos, ou seja, o número de sintomas assinalados em cada quadro e não sua porcentagem.

IHS - É um instrumento que tem como finalidade aferir o repertório de habilidades sociais usualmente requerido em uma amostra de situações 
interpessoais cotidianas. Para tanto, a tarefa do sujeito é fazer uma estimativa da frequência com que reage da forma descrita em cada item, considerando o total de vezes em que se encontrou naquela situação e assinalar sua resposta. O inventário é composto por 38 itens, estruturados em escala likert de cinco pontos (nunca ou raramente, com pouca frequência, com regular frequência, muito frequentemente e sempre ou quase sempre), distribuídos em cinco fatores ou subescalas: enfrentamento com risco $(1,5,7,11,12,14,15,16,20,21 \mathrm{e}$ 29; alfa de Cronbach de 0,96); autoafirmação na expressão de afeto positivo (3, 6, 8, 10, 28, 30 e 35; alfa de Cronbach de 0,86); conversação e desenvoltura social $(13,17,19,22,24,36$ e 37; alfa de Cronbach de 0,81); autoexposição a desconhecidos ou a situações novas (9, 14, 23 e 26; alfa de Cronbach de 0,75); e autocontrole da agressividade a situações aversivas (18, 31 e 38; alfa de Cronbach de 0,74) (Del Prette \& Del Prette, 2001). O alfa de Cronbach de 0,75 para a escala geral é considerado satisfatório. Para minimizar o efeito da "desejabilidade social", 15 itens foram redigidos com fraseado negativo, ou seja, a reação sugerida é um indicativo de falta de habilidade $(2,8,9,13,17,18,19,22,23,24,26$, 33, 34, 36 e 37).

A pontuação do inventário se dá pela soma dos itens assinalados pelos sujeitos, variando de 0 a 152 pontos. Os escores são convertidos em percentil e em resultados $\mathrm{T}$, de acordo com as especificações do manual. Há dados normativos específicos para cada sexo.

\subsection{Procedimento}

Após a obtenção da autorização da direção das instituições, o instrumento foi aplicado, individualmente ou em grupos com no máximo 20 sujeitos, em uma sala no próprio local de trabalho dos profissionais. A coleta de dados foi realizada em horários previamente agendados e somente com aqueles profissionais que assinaram o termo de consentimento livre e esclarecido. A aplicação do instrumento foi realizada pelos próprios pesquisadores e foram necessários 30 minutos, por aplicação, para que todo o procedimento planejado fosse executado.

\section{RESULTADOS E DISCUSSÃO}

Considerando os objetivos deste estudo, foram utilizadas provas de estatística descritiva e inferencial. A tabela $1 \mathrm{traz}$ as estatísticas descritivas relativas ao desempenho dos participantes no ISSL. 
Tabela 1. Estatísticas descritivas dos resultados obtidos no ISSL ( $N=152)$

\begin{tabular}{|l|c|c|c|c|}
\hline \multicolumn{1}{|c|}{ ISSL } & Mínimo & Máximo & Média & DP \\
\hline $\begin{array}{l}\text { Sintomas nas últimas } \\
24 \text { horas }\end{array}$ & 0 & 7 & 1,63 & 1,67 \\
\hline $\begin{array}{l}\text { Sintomas na última } \\
\text { semana }\end{array}$ & 0 & 13 & 1,95 & 2,50 \\
\hline $\begin{array}{l}\text { Sintomas no último } \\
\text { mês }\end{array}$ & 0 & 16 & 2,02 & 2,69 \\
\hline Total & 0 & 27 & 5,61 & 5,87 \\
\hline
\end{tabular}

Fonte: elaborada pelas autoras.

Como pode ser observado na tabela 1 , as maiores pontuações foram obtidas pelos participantes no último mês $(M=2,02 ; \mathrm{DP}=2,29)$ e na última semana $(\mathrm{M}=1,95 ; \mathrm{DP}=2,50)$, respectivamente. Em acréscimo, verificou-se que dos 152 docentes avaliados, apenas $35(23 \%)$ apresentaram sintomas que atingem os limites característicos de estresse. Destes, 33 (94,3\%) apresentaram sintomas característicos da fase de resistência e $2(5,7 \%)$ da fase de quase exaustão. Os sintomas mais frequentes na amostra foram cansaço constante e excessivo $(n=49$; $32,2 \%)$, tensão muscular $(\mathrm{n}=47 ; 30,9 \%)$ e insônia ou dificuldade para dormir $(\mathrm{n}=39 ; 25,7 \%)$. Tais resultados corroboram os dados encontrados por Oliveira e Cardoso (2011), demonstrando uma predominância de sintomas na fase resistência e são parcialmente coerentes com os achados de Pereira, Amaral, e Scorsolini-Comin (2011), tendo em vista que a maioria dos professores com sintomas de estresse (91\%) deste estudo também estavam na fase de resistência, contudo nenhum professor na fase de exaustão foi encontrado.

No que concerne às habilidades sociais, faz-se necessário ressaltar que se procedeu à ponderação dos resultados brutos obtidos no IHS, tendo em vista a não homogeneidade no número de itens que compõem cada um dos seus fatores. Considerando o exposto, os resultados referentes aos cinco fatores da escala e à pontuação total são apresentados na tabela 2. 
Tabela 2. Estatísticas descritivas dos resultados obtidos no IHS ( $N=152)$

\begin{tabular}{|l|c|c|c|c|}
\hline \multicolumn{1}{|c|}{ EST } & Mínimo & Máximo & Média & DP \\
\hline $\begin{array}{l}\text { Fator 1 - Enfrentamento e } \\
\text { autoafirmação com risco }\end{array}$ & 0,00 & 3,73 & 2,52 & 0,72 \\
\hline $\begin{array}{l}\text { Fator 2 - Autoafirmação na expressão } \\
\text { de sentimento positivo }\end{array}$ & 0,57 & 4,00 & 3,10 & 0,62 \\
\hline $\begin{array}{l}\text { Fator 3 - Conversação e desenvoltura } \\
\text { social }\end{array}$ & 0,57 & 4,00 & 3,09 & 0,55 \\
\hline $\begin{array}{l}\text { Fator 4 - Autoexposição a } \\
\text { desconhecidos e situações novas }\end{array}$ & 0,75 & 4,00 & 2,98 & 0,79 \\
\hline $\begin{array}{l}\text { Fator 5 - Autocontrole da } \\
\text { agressividade }\end{array}$ & 0,00 & 3,00 & 1,98 & 0,65 \\
\hline Total & 1,58 & 3,61 & 2,81 & 0,45 \\
\hline
\end{tabular}

Fonte: elaborada pelas autoras.

Os professores obtiveram uma média de 2,81 pontos $(D P=0,45)$ no IHS, com uma pontuação mínima de 1,58 e máxima de 3,61 pontos. No que se refere aos subtestes que compóem a escala, observou-se que, por um lado, as pontuações médias obtidas no "Fator 2 - Autoafirmação na expressão de sentimento positivo" e "Fator 3 - Conversação e desenvoltura social" foram superiores às demais $(M=3,10 ; D P=0,62$ e $M=3,09 ; D P=0,55$, respectivamente), por outro, que os piores desempenhos ocorreram no "Fator 5 - Autocontrole da agressividade" e "Fator 1 - Enfrentamento e autoafirmação com risco" $(M=1,98$; $D P=0,65$ e $M=2,52 ; D P=0,72$, respectivamente). Esses resultados indicam que a amostra pesquisada tem baixo repertório de habilidades sociais para lidar com situações interpessoais em que a demanda de reação ao interlocutor se caracteriza principalmente pela afirmação e defesa de direitos e de autoestima, com risco potencial de reação indesejável por parte do interlocutor e de reações a estimulações aversivas por parte deste, demandando controle da raiva e da agressividade.

Tais resultados são parcialmente coerentes com os encontrados por Soares e Mello (2010), nos quais os professores obtiveram uma média de 2,78 pontos ( $\mathrm{DP}=$ $0,43)$. Já no que se referem aos subtestes, os autores observaram que as pontuaçóes médias obtidas no "Fator 2 - Autoafirmação na expressão de sentimento positivo" e no "Fator 5 - Autocontrole da agressividade" foram superiores às demais e os piores desempenhos ocorreram no "Fator 1- Enfrentamento e autoafirmação com risco" e "4 - Autoexposição a desconhecidos e situações novas". 
Por fim, os resultados referentes à relação entre os construtos podem ser observados na tabela 3. Ressalta-se, no entanto, que tal relação foi verificada com base na comparação das médias obtidas no IHS ao se considerarem grupos extremos ou contrastantes em termos de estresse.

Tabela 3. Comparação entre as pontuações obtidas no IHS em função do ISSL ( $N=152$ )

\begin{tabular}{|c|c|c|c|c|c|c|}
\hline IHS & ISSL & $N$ & Média & $D P$ & $t$ & $p$ \\
\hline \multirow{2}{*}{$\begin{array}{l}\text { Fator } 1 \text { - } \\
\text { Enfrentamento e } \\
\text { autoafirmação com } \\
\text { risco }\end{array}$} & $\begin{array}{c}\text { Sem } \\
\text { estresse }\end{array}$ & 58 & 2,54 & 0,71 & \multirow{2}{*}{0,63} & \multirow{2}{*}{0,530} \\
\hline & $\begin{array}{c}\text { Com } \\
\text { estresse }\end{array}$ & 62 & 2,45 & 0,75 & & \\
\hline \multirow{2}{*}{$\begin{array}{l}\text { Fator } 2 \text { - Autoafirmação } \\
\text { na expressão de } \\
\text { sentimento positivo }\end{array}$} & $\begin{array}{c}\text { Sem } \\
\text { estresse }\end{array}$ & 58 & 3,07 & 0,66 & \multirow{2}{*}{$-1,44$} & \multirow{2}{*}{0,153} \\
\hline & $\begin{array}{c}\text { Com } \\
\text { estresse }\end{array}$ & 62 & 3,21 & 0,43 & & \\
\hline \multirow{2}{*}{$\begin{array}{l}\text { Fator } 3 \text { - Conversação e } \\
\text { desenvoltura social }\end{array}$} & $\begin{array}{c}\text { Sem } \\
\text { estresse }\end{array}$ & 58 & 3,14 & 0,50 & \multirow{2}{*}{1,90} & \multirow{2}{*}{0,063} \\
\hline & $\begin{array}{c}\text { Com } \\
\text { estresse }\end{array}$ & 62 & 2,91 & 0,67 & & \\
\hline \multirow{2}{*}{$\begin{array}{l}\text { Fator } 4 \text { - Autoexposição } \\
\text { a desconhecidos e } \\
\text { situações novas }\end{array}$} & $\begin{array}{c}\text { Sem } \\
\text { estresse }\end{array}$ & 58 & 2,98 & 0,79 & \multirow{2}{*}{$-0,05$} & \multirow{2}{*}{0,964} \\
\hline & $\begin{array}{c}\text { Com } \\
\text { estresse }\end{array}$ & 62 & 2,98 & 0,80 & & \\
\hline \multirow{2}{*}{$\begin{array}{l}\text { Fator } 5 \text { - Autocontrole } \\
\text { da agressividade }\end{array}$} & $\begin{array}{c}\text { Sem } \\
\text { estresse }\end{array}$ & 58 & 1,96 & 0,66 & \multirow{2}{*}{$-0,57$} & \multirow{2}{*}{0,571} \\
\hline & $\begin{array}{c}\text { Com } \\
\text { estresse }\end{array}$ & 62 & 2,03 & 0,61 & & \\
\hline \multirow{2}{*}{ Total } & $\begin{array}{c}\text { Sem } \\
\text { estresse }\end{array}$ & 58 & 2,83 & 0,46 & \multirow{2}{*}{0,97} & \multirow{2}{*}{0,336} \\
\hline & $\begin{array}{c}\text { Com } \\
\text { estresse }\end{array}$ & 62 & 2,75 & 0,40 & & \\
\hline
\end{tabular}

Fonte: elaborada pelas autoras.

Não houve diferenças significativas em habilidades sociais entre os docentes com sintomatologia de estresse e os sem sintomatologia, tal como pode ser observado na tabela. Os professores que obtiveram sintomatologia de estresse apresentaram repertórios mais restritos nos fatores 1 (Enfrentamento $\mathrm{e}$ autoafirmação com risco), 3 (Conversação e desenvoltura social) e na pontuação total do teste, apesar da ausência da significância estatística. Esses resultados, 
por um lado, estão em dissonância com os pressupostos teóricos vigentes, uma vez que se esperava que os profissionais que apresentam níveis de estresse mais elevados fossem aqueles com um menor repertório de habilidades sociais. Por outro, convergem com estudos, quer com professores, ou não professores, que não têm encontrado associações significativas entre estresse e habilidades sociais (Gomes et al., 2013; Grün, 2008; Pellegrini, Calais, \& Salgado, 2012).

\section{CONSIDERAÇÕES FINAIS}

O trabalho do professor envolve diferentes aspectos que perpassam desde a seleção de conteúdos, organização de programas, escolha de metodologias adequadas para o ensino, acompanhamento da aprendizagem do aluno, gestão da classe, estrutura organizacional, pressupostos, valores, diferentes entendimentos das políticas educacionais, condições de trabalho, opções didáticas, organização e âmbito das atividades, organização do tempo e do espaço até a interação com diferentes agentes, sejam eles alunos, pais, pares, entre outros. Logo, apresentar comportamentos que facilitem os relacionamentos interpessoais pode favorecer o exercício da profissão, o manejo de situações ou eventos estressores e a permanência do docente no trabalho.

Dessa forma, tendo em vista a relevância dos temas expostos e considerandose o impacto do estresse e das habilidades sociais na vida dos indivíduos, bem como a importância da avaliação psicológica mediante o uso de instrumentos com parâmetros psicométricos adequados, esta pesquisa contribuiu para aprofundar os estudos na área de habilidades sociais e estresse, bem como trouxe contribuiçõos para a identificação dessas variáveis no processo de trabalho dos docentes em instituições públicas e privadas de cidades localizadas no Estado da Bahia, no Nordeste do Brasil. Espera-se que os resultados obtidos possam agregar conhecimentos e contribuir para o avanço científico também da área de avaliação, além de poder subsidiar possíveis práticas voltadas para determinadas categorias profissionais, como a categoria docente. Faz-se necessário ressaltar, ainda, a importância do desenvolvimento de outras pesquisas que visem a estudar não apenas os construtos aqui focalizados, mas os parâmetros psicométricos dos instrumentos empregados, bem como sua relação com outras variáveis, a fim de sanar as possíveis lacunas deixadas por este estudo.

Apesar do baixo índice de docentes com estresse na amostra investigada, ressalta-se a importância de que esses quadros sejam acompanhados e cuidados para que não evoluam e caracterizem condições de exaustão emocional intensa ou passem a ter impactos ainda mais significativos nas condições físicas e psicológicas desses profissionais, afetando, assim, sobremaneira, suas relações interpessoais 
e qualidade de vida. Em que pesem as limitações desta pesquisa, tais como o número reduzido de participantes, instituiçôes e a quantidade ainda restrita de estudos voltados para a avaliação conjunta do estresse e das habilidades sociais em docentes e que possibilitem maior respaldo para seus achados, considera-se que estudos com um maior aprofundamento devem ser realizados levando em consideração as possibilidades de investigação citadas anteriormente.

Pesquisas desse porte são relevantes não apenas por possibilitarem o desenho e o desenvolvimento da área, mas, sobretudo, porque funcionam como um indicativo das lacunas que precisam ser preenchidas, contribuindo, assim, para a disseminação, a qualidade e progresso do conhecimento produzido nas academias. A partir deste estudo, pode-se pensar em programas tanto de manejo do estresse quanto de desenvolvimento de habilidades sociais, que promovam uma maior qualidade nas interaçóes dos profissionais do magistério, possibilitando que estes estejam aptos a exercerem seu papel fundamental, que é formar, para além da apreensão de conteúdos e comprovação destes, cidadãos aptos a exercerem seus deveres e direitos em qualquer nível de ensino. 


\section{REFERÊNCIAS}

Bachion, M. M., Abreu, L. O. de, Godoy, L. F. \& Costa, E. C. (2005). Vulnerabilidade ao estresse entre professores de uma universidade pública. Revista Brasileira de Enfermagem UERJ, 13, 32-37.

Bandeira, M., Rocha, S. S., Pires, L. G., Del Prette, Z. A. P. \& Del Prette, A. (2006). Competência acadêmica de crianças do ensino fundamental: características sociodemográficas e relação com habilidades sociais. Interação em Psicologia, 10(1), 53-62.

Bighetti, C. A. (2010). Escala de depressão (Edep) e sua relação com medidas de personalidade e habilidades sociais. (Tese de Doutorado). Universidade São Francisco, Programa de Pós-Graduação em Psicologia, Itatiba.

Borine, B., Assis, C. L., Lopes, M. S. \& Santini, T. O. (2012). Estresse hospitalar em equipe multidisciplinar de hospital público do interior de Rondônia. Revista da SBPH, 15(1), 22-40.

Carneiro, R. S. \& Falcone, E. M. O. (2013). O desenvolvimento das habilidades sociais em idosos e sua relação na satisfação com a vida. Estudos de Psicologia, 18(3), 517-526.

Christophoro, R. \& Waidman, M. A. P. (2002). Estresse e condições de trabalho: um estudo com docentes do curso de enfermagem da UEM, Estado do Paraná. Acta Scientiarum, 24(3), 757-763.

Costa, M. F. A. A. \& Ferreira, M. C. (2014). Sources and reactions to stress in Brazilian Lawyers. Paidéia, 24(57), 49-56.

Esteves, M. F. D. P. (2004). Estresse psíquico em professores do ensino superior privado: um estudo em Salvador - BA. (Dissertação de Mestrado). Universidade Federal da Bahia, Programa de Pós-Graduação em Psicologia, Salvador.

Fernandes, S. (1999). Transformações no mundo do trabalho e a saúde psíquica: a ótica do estresse ocupacional. Organizaçôes \& Sociedade, 6(16), 67-75.

Gomes, A. S., Pereira, J. W. S., Viveiros, J. O., Mendes, J., Gabriel, M. A. O, Assis, M. R., ... Nascimento, V. L. (2013). Habilidades sociais e estresse: um estudo com professores do ensino fundamental de escolas públicas. Desenvolvimento Humano Revista Eletrônica, 12(4), 719-731. 
Gomide, P. I. C., Salvo, C. G. de, Pinheiro, D. P. N. \& Sabbag, G. M. (2005). Correlação entre práticas educativas, depressão, estresse e habilidades sociais. Psico-USF, 10(2), 169-178.

Grün, T. S. (2008). Stress e habilidades sociais em pacientes com câncer de laringe. (Dissertação de Mestrado). Pontifícia Universidade Católica de Campinas, Programa de Pós-Graduação em Psicologia, Campinas.

Lipp, M. E. N. (2000). Manual do inventário de sintomas de stress para adultos de Lipp (ISSL). São Paulo: Casa do Psicólogo.

Lipp, M. E. N. (2008). Estudos sobre o stress. Instituto de Psicologia e Controle do Stress Marilda Emmanuel Novaes Lipp. Recuperado a partir de http://www. estresse.com.br.

Lucca, E. (2004). Habilidade social: uma questão de qualidade de vida. Psicologia: o portal dos psicólogos. Recuperado a partir de http://www.psicologia.com.pt/ artigos/textos/A0224.pdf

Maia, D. da S. \& Bortolini, M. (2012). O desenvolvimento da habilidade de assertividade e a convivência na escola: relato de experiência. Psicologia em Revista, 18(3), 373-388.

Maia, D. da S. \& Lobo, B. de O. M. (2013). O desenvolvimento da habilidade de solução de problemas interpessoais e a convivência na escola. Psicologia em Revista, 19(1), 17-29.

Malagris, L. E. N. \& Fiorito, A. C. C. (2007). Avaliação do nível de stress de técnicos da área de saúde. Estudos de Psicologia, 23(4), 391-398.

Minari, M. R. T. \& Souza, J. C. (2011). Stress em servidores públicos do instituto nacional de seguro social. Estudos de Psicologia, 28(4), 521-528.

Oliveira, M. G. M. \& Cardoso, C. L. (2011). Stress e trabalho docente na área de saúde. Estudos de Psicologia Campinas, 28(2), 135-141.

Pellegrini, C. F. S., Calais, S. L. \& Salgado, M. H. (2012). Habilidades sociais e administração de tempo no manejo do estresse. Arquivos Brasileiros de Psicologia, 64(3), 110-129.

Pereira, H. O. S. de, Amaral, M. C. do. \& Scorsolini-Comin, F. (2011). Avaliação de sintomas de estresse em professores universitários: qualidade de vida no fazer docente. Educação: Teoria e Prática, 21(37), 71-91. 
Del Prette, Z. A. P. \& Del Prette, A. (2001). Inventário de Habilidades Sociais (IHS-Del-Prette): manual de aplicação, apuração e interpretação. São Paulo: Casa do Psicólogo.

Del Prette, Z. A. P. \& Del Prette, A. (2010/2012). Social skills and behavior analysis: historical proximity and new Issues. Perspectivas em Análise do Comportamento, 1(2), 104-115.

Del Prette, Z. A. P., Del Prette, A. \& Barreto, M. C. M. (2006). Treinamento de habilidades sociais em grupo com estudantes de Psicologia: avaliando um programa de intervenção. In M. Bandeira, Z. A. P. Del Prette \& A. Del Prette (Orgs.), Estudos sobre habilidades sociais e relacionamento interpessoal. (pp. 217234). São Paulo: Casa do Psicólogo.

Pureza, J. R., Rursch, S. G. S., Wagner, M. \& Oliveira, M. S. (2012). Treinamento de habilidades sociais em universitários: uma proposta de intervenção. Revista Brasileira de Terapias Cognitivas, 8(1), 2-9.

Reinhold, H. H. (1996). Stress ocupacional do professor. In M. E. N. Lipp (Org.), Pesquisas sobre stress no Brasil: saúde, ocupaçôes e grupos de risco. (pp. 169-194). Campinas: Papirus.

Reis, E. J. F., Carvalho, F. M., Araújo, T. M., Porto, L. A. \& Silvany Neto, A. M. (2005). Trabalho e distúrbios psíquicos em professores da rede municipal de Vitória da Conquista, Bahia, Brasil. Cadernos de Saúde Pública, 21(5), 14801490 .

Rockhill, C., Stoep, A. V., Mccauley, E. \& Katon, W. J. (2009). Social competence and social support as mediators between comorbid depressive and conduct problems and functional outcomes in middle school students. J Adolesc, 32(3), $535-553$.

Sadir, M. A., Bignotto, M. M. \& Lipp, M. E. N. (2010). Stress e qualidade de vida: influência de algumas variáveis pessoais. Paideia, 20(45), 73-81.

Sadir, M. A. \& Lipp, M. E. N. (2013). Influência do treino de controle do estresse nas relações interpessoais no trabalho. O mundo da saúde, 37(2), 131140 .

Santos, A. F. O. \& Cardoso, C. L. (2010). Profissionais de saúde mental: estresse, enfrentamento e qualidade de vida. Psicologia: Teoria e Pesquisa, 26(3), 543548. 
Segantin, B. G. O \& Maia, E. M. F. L. (2007). Estresse vivenciado pelos profissionais que trabalham na saúde. Recuperado a partir de http://www.inesul.edu.br/ revista/arquivos/arq-idvol_5_1247866839.pdf

Servilha, E. A. M. (2005). Estresse em professores universitários na área de fonoaudiologia. Revista Ciências Médicas, 14(1), 43-52.

Soares, A. B., Gomes, G. \& Prata, M. A. R. (2011). Habilidades sociais de professores e não professores: comparando áreas de atuação. Revista Colombiana de Psicologia, 20(2), 233-248.

Soares, A. B. \& Mello, T. V. S. (2010). Habilidades sociais entre professores e não professores. Revista Brasileira de Terapias Cognitivas, 5(2), 15-27.

Takahashi, L. T. (2009). Avaliação da vulnerabilidade ao estresse no trabalho de operadores de telemarketing por meio da EVENT. (Dissertação de Mestrado). Universidade São Francisco, Programa de Pós-Graduação em Psicologia, Itatiba.

Tamayo, A. (2001). Prioridades axiológicas, atividade física e estresse ocupacional. Revista de Administração Contemporânea, 5(3), 127-147.

Tironi, M. O. S., Nascimento Sobrinho, C. L., Barros, D. S., Reis, E. J. F. B., Marques Filho, E. S., Almeida, A., ... \& Souza, Y. M. (2009). Trabalho e síndrome da estafa profissional (Síndrome de Burnout) em médicos intensivistas de Salvador. Revista da Associação Médica Brasileira, 55(6), 656-662. 